\title{
ALK expression in extranodal anaplastic large cell lymphoma favours systemic disease with (primary) nodal involvement and a good prognosis and occurs before dissemination
}

Rosita L ten Berge, Joost J Oudejans, Gert-Jan Ossenkoppele, Karen Pulford, Rein Willemze, Brunangelo Falini, Andreas Chott, Chris J L M Meijer

\begin{abstract}
Aims-In anaplastic large cell lymphoma (ALCL), the site of origin has been described as an important prognostic factor. Recently, a fusion protein containing anaplastic lymphoma kinase (ALK) was described in systemic nodal ALCL, and shown to be associated with a good prognosis. The aims of this study were to investigate whether the presence of ALK protein differs between ALCL of different sites of origin; to determine whether ALK expression occurs before dissemination to other sites; and, finally, to investigate whether the site of origin remains a prognostic parameter in ALK negative ALCL. Methods-ALK expression, as detected by immunohistochemistry using the monoclonal antibodies ALK1 and ALKc, was studied in 85 ALCLs from different sites of origin. In 22 patients, ALK expression was studied in multiple biopsies from different sites (including 13 skin, 16 lymph node, and nine other). Overall survival time was analysed using the Kaplan Meier method.
\end{abstract} Results-ALK expression was found in 20 of 51 systemic ALCLs with (primary) nodal involvement. No ALK expression was found in 15 primary cutaneous, 14 gastrointestinal, and five nasal ALCLs. Multiple and subsequent biopsies of patients showed ALK expression to be identical to that seen in the primary diagnostic biopsy. Kaplan Meier survival curves showed that in ALK negative ALCLs originating from different sites, primary cutaneous cases are associated with an excellent overall survival, whereas the other cases show a comparable five years survival of less than $40 \%$.

Conclusions-If present, ALK expression favours systemic ALCL with (primary) nodal involvement, and can be used in differentiating between extranodal involvement of systemic (nodal) ALCL and primary extranodal ALCL. ALK is expressed consistently in multiple biopsies of a given patient, indicating that the chromosomal abnormality leading to aberrant ALK expression occurs before dissemination to other sites. Finally, in ALK negative non-cutaneous ALCLs, different sites of origin show comparable poor survival.

(f Clin Pathol 2000;53:445-450)
Keywords: anaplastic large cell lymphoma; extranodal; anaplastic lymphoma kinase; survival

In 1985, anaplastic large cell lymphoma (ALCL) was recognised as a separate disease entity, ${ }^{1}$ characterised by cohesive sheets of large CD30 positive cells with multiple or single prominent nucleoli and $\mathrm{T}$ cell or null cell characteristics. ${ }^{2}$ In 1993, we described differences in clinical behaviour between primary nodal and primary cutaneous ALCL. ${ }^{3}$ These differences are reflected in the two types of ALCL recognised in the revised EuropeanAmerican classification of lymphoid neoplasms (REAL classification). ${ }^{2}$ The first type is a systemic variant, mainly presenting in lymph nodes, with a prognosis better than that of other T cell lymphomas. ${ }^{4}$ The second is a primary cutaneous variant with a very favourable prognosis. ${ }^{356}$ Recently, at the IX meeting of the European Association for Haematopathology (Leiden, The Netherlands, April 26-29, 1998) the proposed list for the new WHO classification of leukaemias and lymphomas was presented. This included among the extranodal $\mathrm{T}$ cell lymphomas the enteropathy type intestinal $\mathrm{T}$ cell lymphoma and the (natural killer/T cell) nasal type, which are usually very aggressive, irrespective of whether their morphology is small cell or anaplastic. ${ }^{2-9}$ Thus, in the group of lymphomas with anaplastic morphology, distinction between different sites of origin is important, because of the differences in clinical behaviour and consequent therapeutic strategies.

About $15-85 \%$ of systemic (nodal) ALCLs contain the $\mathrm{t}(2 ; 5)(\mathrm{p} 23 ; \mathrm{q} 35)$ translocation, which fuses the anaplastic lymphoma kinase (ALK) gene at 2p23 with the nucleophosmin (NPM) gene at 5q35, resulting in a fusion protein NPM-ALK. ${ }^{10}$ In about $15 \%$ of ALK positive cases, genetic aberrations other than the $t(2 ; 5)$ translocation are involved, fusing the ALK gene to a gene other than NPM. ${ }^{11-16}$ Recently, two monoclonal antibodies, ALK $1^{15}$ and ALKc, ${ }^{17}$ were developed that detect the ALK portion of the fusion protein in formalin fixed, paraffin wax embedded tumour specimens by immunohistochemistry. Previous to that, the polyclonal antibody p 80 had been developed. ${ }^{18}$ In various studies, expression of ALK was found to be related to a more favourable clinical outcome. ${ }^{19-23}$ Expression of $t(2 ; 5)$ and/or ALK in ALCLs at different sites has been studied by several groups, their results 
Table 1 ALK expression in anaplastic large cell lymphoma (ALCL) according to site of origin

\begin{tabular}{|c|c|c|c|c|c|}
\hline & \multirow[b]{2}{*}{$N$} & \multirow[b]{2}{*}{ ALK expression } & \multicolumn{3}{|c|}{ Pattern of staining } \\
\hline & & & $c / n$ & $c$ & $n$ \\
\hline Systemic (nodal) ALCL & 51 & 20 & 15 & 5 & 0 \\
\hline Primary gastrointestinal ALCL & 14 & 0 & - & - & - \\
\hline Primary nasal ALCL & 5 & 0 & - & - & - \\
\hline \multicolumn{6}{|l|}{ Cutaneous CD30+ LPD } \\
\hline Primary cutaneous ALCL & 15 & 0 & - & - & - \\
\hline Lymphomatoid papulosis & 7 & 0 & - & - & - \\
\hline
\end{tabular}

ALK expression was determined by immunohistochemistry using the monoclonal antibodies ALK1 and ALKc.

$\mathrm{c} / \mathrm{n}$, cytoplasmic and nuclear staining; $\mathrm{c}$, cytoplasmic staining only; $\mathrm{n}$, nuclear staining only; LPD, lymphoproliferative diseases.

depending on the detection methods used. Using cytogenetics, reverse transcriptase polymerase chain reaction (RT-PCR), in situ hybridisation (ISH), and immunohistochemistry, most groups found ALK expression in systemic (nodal) ALCL and not in primary cutaneous ALCL or lymphomatoid papulosis. ${ }^{1524-26}$ However, in two studies NPM-ALK transcripts were detected in cutaneous ALCL by nested RT-PCR with two amplification steps. ${ }^{27}{ }^{28}$ Another study ${ }^{29}$ found positive staining with the antibodies p 80 and ALK1 in one of eight cases of primary cutaneous ALCL, but no evidence of ALK expression with standard RT-PCR. To our knowledge, ALK expression has not been studied in nasal $\mathrm{T}$ cell lymphomas with anaplastic morphology. A recent study by Ott and colleagues ${ }^{27}$ found no evidence of the $t(2 ; 5)$ translocation in two primary gastrointestinal ALCLs; the study on ALK positive ALCLs by Benharroch and colleagues ${ }^{30}$ included 18 extranodal samples, but did not mention whether these samples included primary gastrointestinal ALCLs.
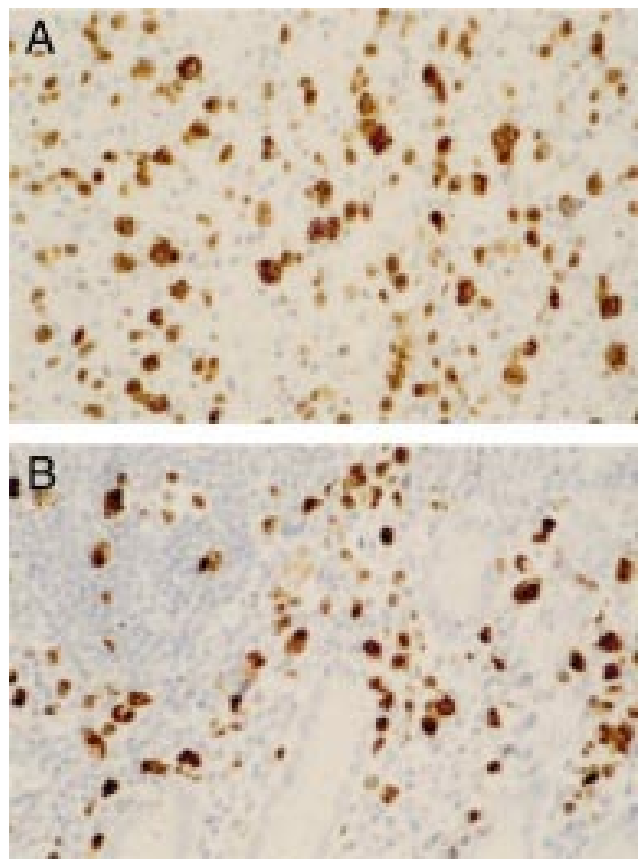

Figure 1 Anaplastic lymphoma kinase (ALK) expression in tumour biopsies of a patient with concurrent nodal $(A)$ and intestinal (B) anaplastic large cell lymphoma. Brown cytoplasmic and nuclear staining of the tumour cells indicates ALK expression, as detected by the monoclonal antibody $A L K c$. Haematoxylin counterstaining; original magnification, $\times 400$.
In our present study, we investigated the expression of ALK in ALCLs arising at different sites, and whether ALK expression can be used to distinguish between systemic (nodal) ALCL and primary extranodal ALCL. Furthermore, the expression of ALK was studied in multiple and/or subsequent tumour biopsies of patients, to determine whether the $\mathrm{t}(2 ; 5)$ translocation is an early pathogenic eventbefore dissemination to other sites. Finally, we investigated whether the site of origin in ALK negative ALCL remains an important prognostic parameter.

\section{Methods}

PATIENT SELECTION

From the files of the Comprehensive Cancer Center Amsterdam, the Netherlands; and from the department of pathology of the University of Vienna, Austria, we selected patients with systemic (nodal) ALCL $(\mathrm{n}=51)$, including morphological subtypes such as the small cell variant $(n=4) .^{30} 31$ Furthermore, primary cutaneous CD30 positive lymphoproliferative disorders were selected, including primary cutaneous ALCL $(\mathrm{n}=15)$ and lymphomatoid papulosis $(n=7)$. We also selected primary gastrointestinal $(n=14)$ and nasal $(n=5) T$ cell lymphomas with anaplastic morphology, consisting of large tumour cells with pleomorphic or monomorphic round nuclei, and multiple or single prominent nucleoli. These cases are henceforward referred to in the text as primary gastrointestinal ALCL and primary nasal ALCL. All samples showed CD30 expression of virtually all tumour cells. Moreover, they were of $\mathrm{T}$ cell lineage (they expressed CD3 and/or CD45RO/UCHL-1, in the absence of B cell markers L26/CD20 and CD79a) or of null cell lineage (both $\mathrm{T}$ and $\mathrm{B}$ cell markers were absent).

Systemic (nodal) ALCL was defined as disease mainly involving lymph nodes, with or without extranodal tumour localisations. $\mathrm{Pa}-$ tients showing coexistent nodal and extranodal localisations were categorised as systemic (nodal) ALCL if the lymph node localisations were not restricted to nodes draining the extranodal tumour. This included one patient who presented with intestinal (jejunal) ALCL concurrent with nodal (mesenteric and paraaortic) localisations.

In contrast, ALCLs that presented clinically primarily in the nose or in the gastrointestinal tract (with no or only locoregional lymph node involvement), were defined as primary nasal and primary gastrointestinal ALCLs, respectively. ALCLs arising in the skin were considered primary cutaneous if extensive staging of patients showed no extracutaneous disease within six months of diagnosis.

From 22 patients, we obtained multiple biopsies from concurrent localisations $(n=7)$ or relapses $(\mathrm{n}=31)$, including 13 skin localisations, 16 nodal, and nine other localisations. For each patient the following characteristics were noted from the medical records: age at diagnosis, sex, Ann Arbor stage at presentation, treatment, response, the occurrence of relapses, and cause of death. 

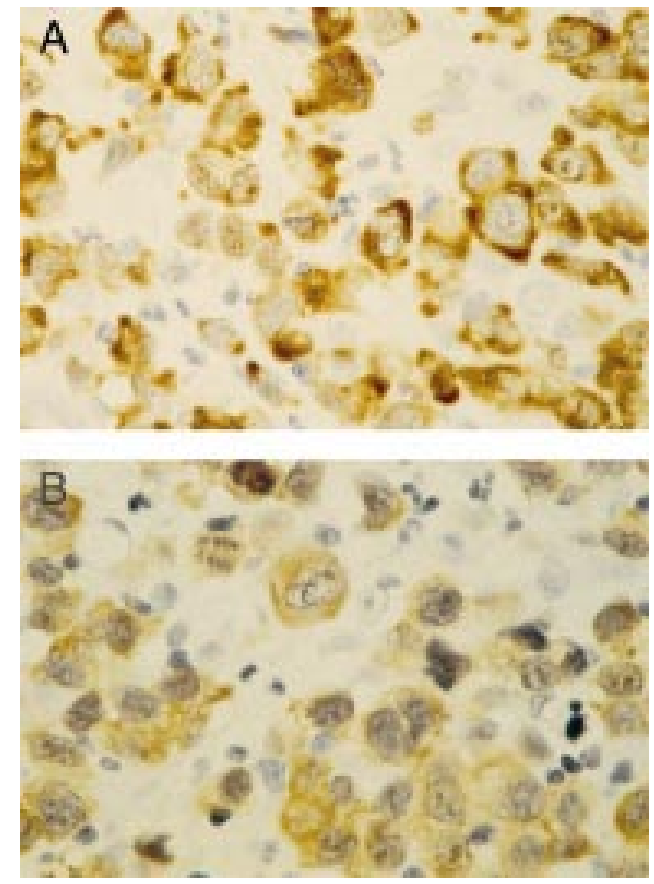

Figure 2 A systemic (nodal) anaplastic large cell lymphoma case with brown, cytoplasm restricted anaplastic lymphoma kinase (ALK) staining of the tumour cells. The cytoplasm restricted staining pattern is the same for monoclonal antibodies ALK1 $(A)$ and $A L K c$ (B).

Haematoxylin counterstaining; original magnification, $\times 600$.

ALK DETECTION

Expression of ALK was detected in formalin fixed, paraffin wax embedded biopsy specimens by immunohistochemistry using the monoclonal antibodies ALK1 and ALKc,

Table 2 ALK expression in ALCL specimens of patients with multiple biopsies or recurrent disease

\begin{tabular}{|c|c|c|c|c|c|c|c|c|c|c|}
\hline \multirow{2}{*}{\multicolumn{3}{|c|}{ Site of origin }} & \multirow{2}{*}{\multicolumn{2}{|c|}{$\begin{array}{l}\text { Colocalisation at } \\
1 \text { st presentation }\end{array}$}} & \multicolumn{6}{|c|}{ Relapse } \\
\hline & & & & & \multicolumn{2}{|l|}{$1 s t$} & \multicolumn{2}{|l|}{$2 n d$} & \multicolumn{2}{|l|}{$3 r d$} \\
\hline Case & Age/sex & $A L K$ & Site & $A L K$ & Site & $A L K$ & Site & $A L K$ & Site & $A L K$ \\
\hline \multicolumn{11}{|c|}{ Systemic (nodal) } \\
\hline 1 & $7 / \mathrm{M}$ & + & & & $\mathrm{LN}$ & + & & & & \\
\hline 2 & $24 / \mathrm{F}$ & + & & & Skin & + & & & & \\
\hline 3 & $24 / M$ & + & & & Skin & + & $\mathrm{LN}$ & + & $\mathrm{LN}$ & + \\
\hline 4 & $29 / \mathrm{F}$ & + & $\mathrm{GI}^{\star}$ & + & & & & & & \\
\hline 5 & $52 / \mathrm{M}$ & - & Skint & - & & & & & & \\
\hline 6 & $18 / \mathrm{M}$ & - & Lungt & - & & & & & & \\
\hline 7 & $68 / \mathrm{F}$ & - & Livert & - & & & & & & \\
\hline 8 & $55 / \mathrm{M}$ & - & & & $\mathrm{LN}$ & - & & & $\mathrm{LN}$ & - \\
\hline 9 & $27 / M$ & - & & & $\mathrm{LN}$ & - & $\mathrm{LN}$ & - & & \\
\hline 10 & $63 / \mathrm{M}$ & - & & & Oes & _- & & & & \\
\hline 11 & $13 / \mathrm{F}$ & - & & & LN & - & & & & \\
\hline \multicolumn{11}{|c|}{ Gastrointestinal } \\
\hline 12 & $51 / \mathrm{M}$ & - & $\mathrm{LN} \ddagger$ & - & Lung & - & & & & \\
\hline 13 & $80 / \mathrm{M}$ & - & $\mathrm{LN} \ddagger$ & - & Bone & - & & & & \\
\hline \multicolumn{11}{|c|}{ Nasal } \\
\hline 14 & $76 / \mathrm{M}$ & - & Testis & - & Bone & - & $\mathrm{LN}$ & - & & \\
\hline 15 & $47 / \mathrm{M}$ & - & & & $\mathrm{LN}$ & - & Bone & - & $\mathrm{LN}$ & - \\
\hline \multicolumn{11}{|c|}{ Cutaneous } \\
\hline 16 & $32 / \mathrm{M}$ & - & Skin & - & & & & & & \\
\hline 17 & $69 / \mathrm{M}$ & - & & & Skin & - & Skin & - & $\mathrm{LN}$ & - \\
\hline 18 & $80 / \mathrm{M}$ & - & & & Skin & - & & & & \\
\hline 19 & $69 / \mathrm{F}$ & - & & & $\mathrm{LN}$ & - & & & & \\
\hline 20 & $29 / \mathrm{F}$ & - & & & Skin & - & Skin & - & Skin & - \\
\hline 21 & $42 / \mathrm{M}$ & - & & & Skin & - & $\mathrm{LN}$ & - & & \\
\hline 22 & $40 / M$ & - & & & Skin & - & Skin & - & & \\
\hline
\end{tabular}

Age is given in years.

*This patient showed concurrent nodal (mesenteric and para-aortic) and intestinal (jejunal) disease. †Nodal involvement was not restricted to lymph nodes draining the extranodal tumour localisations.

$\ddagger$ Locoregional lymph nodes.

M, male; F, female; GI, gastrointestinal; LN, lymph node; Oes, oesophagus.

Note: pattern of ALK staining (cytoplasmic and nuclear, or cytoplasmic only) as demonstrated by the primary biopsy is identical in biopsies from colocalisations or relapses of the same patient. which were developed in the laboratories of KP (Oxford, UK) and BF (Perugia, Italy), respectively. Both antibodies react specifically with an intracytoplasmic epitope of the ALK protein, and correlate strongly with genetic aberrations involving the ALK gene at $2 \mathrm{p} 23 .{ }^{15}{ }^{17}$ Staining was performed as described previously, ${ }^{15} 17$ with minor modifications. Antigen retrieval was achieved by placing dewaxed slides in citrate buffer (0.01 M, pH 6.0) and heating them in a $700 \mathrm{~W}$ microwave oven for 10 minutes. The slides were then removed and allowed to cool at room temperature for 30 minutes. After washing in water, endogenous peroxidase was blocked with $1 \%$ hydrogen peroxide in methanol for 30 minutes, after which slides were rinsed in phosphate buffered saline before beginning the three step staining technique using the DAKO Strept ABC complex/HRP Duet kit. Slides were incubated with ALK1 (using a 1/50 dilution) or ALKc ( $1 / 5$ dilution) for one hour at room temperature, and staining was enhanced by the catalysed reporter deposition (CARD) method, which amplifies biotinylated sites. ${ }^{32}$ The peroxidase reaction was developed using 3,3'-diaminobenzidine tetrahydrochloride (DAB) and $0.01 \%$ vol/vol hydrogen peroxide. There were no discrepancies found between ALK1 and ALKc with regard to presence or absence of staining - if a tumour biopsy stained positively with ALK1 it was also found to be positive with ALKc, and vice versa. Cases were considered ALK positive if tumour cells showed positive labelling, irrespective of their number.

\section{STATISTICAL ANALYSIS}

Survival time was measured from time of initial diagnosis until death as a result of the disease, or until end of follow up. Patients who died of causes unrelated to the disease were censored at the time of death. Progression free survival time was measured from time of initial diagnosis until time of disease relapse.

Survival curves were constructed with the Kaplan Meier method, and differences between the curves were analysed using the log rank test. Comparisons of means were performed using the Kruskal-Wallis test, and qualitative variables were analysed by the Pearson $\chi^{2}$ or Fisher's exact test, where appropriate. All $\mathrm{p}$ values are based on two tailed analysis and $\mathrm{p}$ values below 0.05 were considered to be significant. All analyses were performed using the SPSS statistical software (SPSS Inc Chicago, Illinois, USA).

\section{Results}

ALK EXPRESSION IN SYSTEMIC NODAL AND EXTRANODAL ALCL

As shown in table 1, ALK expression was found in 20 of $51(39.2 \%)$ systemic ALCLs with nodal involvement, including the patient in whom ALCL in an intestinal (jejunal) localisation was found concurrently with abdominal lymph node involvement (fig 1). Of 15 primary cutaneous ALCLs, seven lymphomatoid papulosis, 14 primary gastrointestinal, and 

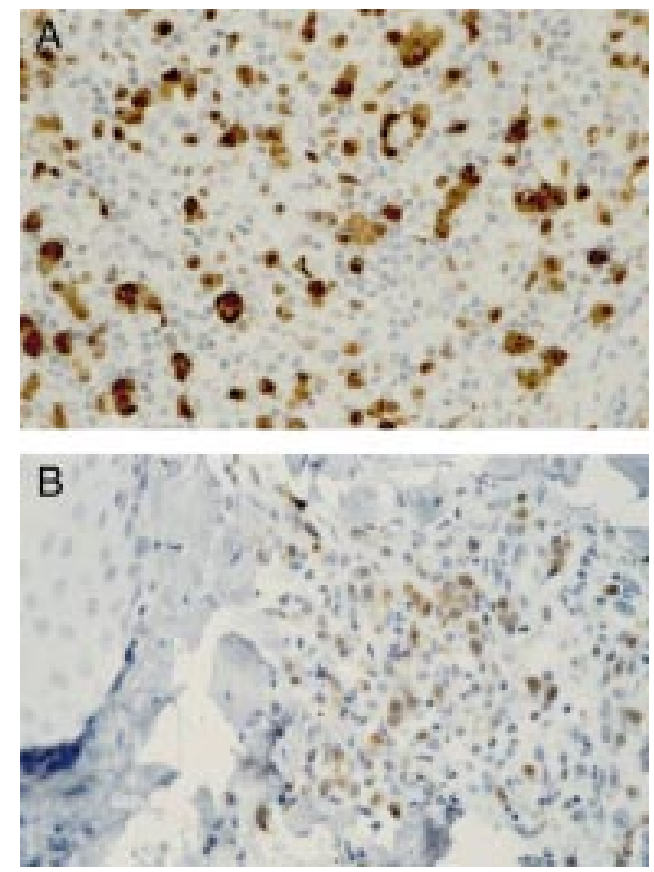

Figure 3 Anaplastic lymphoma kinase (ALK) expression in tumour biopsies of a patient with systemic nodal anaplastic large cell lymphoma $(A)$ and a cutaneous relapse (B). Brown cytoplasmic and nuclear staining of the tumour cells indicates ALK expression, as detected by the monoclonal antibody ALK1. Haematoxylin counterstaining; original magnification, $\times 400$.

five primary nasal ALCLs, none showed ALK expression.

Samples staining positively for ALK1 were also ALKc positive, and vice versa. When present, ALK expression was seen as brown cytoplasmic and nuclear staining of almost all tumour cells in most cases (15 of 20). In five cases, staining was restricted to the cytoplasm of tumour cells (table 1 and fig 2). ALK1 and ALKc antibodies gave the same staining pattern (cytoplasmic and nuclear as opposed to cytoplasmic only), but nuclear positivity tended to be stronger with ALKc.

PRESENCE OR ABSENCE OF ALK EXPRESSION IS CONSISTENT WITHIN PATIENTS

Of the patients with systemic (nodal) ALCL, 11 had multiple biopsies taken at diagnosis and/or presented with recurrent disease. The same was true for seven patients with primary cutaneous ALCL, two with primary gastrointestinal ALCL, and two with primary nasal ALCL (table 2).

ALK expression was found to be consistent within patients; that is, if a patient presented with ALK positive ALCL, all other tumour biopsies of that patient were also found to be ALK positive. For instance, three patients with
ALK positive systemic (nodal) ALCL had one or several relapses (including cutaneous and nodal localisations). ALK positivity was found in all relapses (table 2, cases 1-3; fig 3). In the patient who presented with concurrent intestinal and nodal ALCL, both tumour biopsies showed ALK positivity (table 2 , case 4 ; fig 1 ). The pattern of staining (cytoplasmic and nuclear, or cytoplasmic only), as demonstrated by the primary diagnostic biopsy, remained the same in subsequent biopsies. Finally, in patients presenting with ALK negative lymphoma, all other biopsies of those patients were also found to be ALK negative (table 2).

PATIENT CHARACTERISTICS AND SURVIVAL

We analysed the clinical behaviour of ALCL originating from different primary sites. A full evaluation of clinical data was possible in 48 cases of systemic (nodal) ALCL (18 ALK positive, 30 ALK negative), 10 cases of primary cutaneous ALCL, 13 cases of primary gastrointestinal, and all cases $(n=5)$ of primary nasal ALCL. We found that ALK positive systemic (nodal) ALCL tended to present with Ann Arbor stage 1 or stage 2 (12 of 18 cases), whereas ALK negative systemic (nodal) ALCL presented more often with stage 3 or 4 (20 of 30 cases) (Fisher's exact test, $\mathrm{p}=0.03$ ). Primary cutaneous ALCL usually presented as a solitary skin tumour $(n=12)$, whereas only a few primary cutaneous ALCLs presented as multicentric $(n=2)$ or generalised $(n=1)$ cutaneous tumours. Primary gastrointestinal ALCL was usually confined to the gastrointestinal tract, but in three of 14 cases showed involvement of one other site, such as the spleen (one case; no biopsy available) or a regional lymph node (table 2, cases 12 and 13). Two of five primary nasal ALCLs were confined to the nose, two had locoregional lymph node involvement at the time of diagnosis (no biopsies available), and one case showed involvement of a testicle (table 2, case 14).

In the group of systemic (nodal) ALCLs, ALK expression showed a predilection for younger patients and was significantly related to a favourable clinical outcome (table 3). Only two of 18 patients with ALK positive systemic nodal ALCL (one of whom refused treatment) died as a result of the disease. The patient with concurrent intestinal and nodal ALCL entered complete remission after polychemotherapy, and was alive and well (without relapse) 14 months after diagnosis.

Primary cutaneous ALCL was found to have an excellent prognosis, whereas primary gastrointestinal ALCL showed a five year survival of only $30 \%$ (table 3 ), with more than half the patients dying within one year (fig 4).

Table 3 Clinical characteristics of anaplastic large cell lymphoma of different sites of origin

\begin{tabular}{|c|c|c|c|c|c|c|}
\hline & \multicolumn{2}{|l|}{ Lymph node } & \multirow[b]{2}{*}{ Skin $(n=10)$} & \multirow[b]{2}{*}{$\begin{array}{l}\text { Gastrointestinal } \\
\text { tract }(n=13)\end{array}$} & \multirow[b]{2}{*}{ Nose $(n=5)$} & \multirow[b]{2}{*}{$p$ Value } \\
\hline & $\begin{array}{l}\text { ALK positive } \\
(n=18)\end{array}$ & $\begin{array}{l}\text { ALK negative } \\
(n=30)\end{array}$ & & & & \\
\hline Median age (range) & $25(2-69)$ & $53.5(13-82)$ & $52(31-80)$ & $62(22-80)$ & $76(39-86)$ & $0.003^{\star}$ \\
\hline Died of disease & 2 & 18 & 0 & 9 & 3 & $0.0003^{\star \star}$ \\
\hline Five year survival (\%) & 90 & 35 & 100 & 30 & 0 & $0.0003^{\star \star \star}$ \\
\hline
\end{tabular}

*As determined by the Kruskal-Wallis test; ${ }^{\star \star}$ as determined by the log rank test; ${ }^{\star \star \star}$ as estimated from the Kaplan Meier curve. 


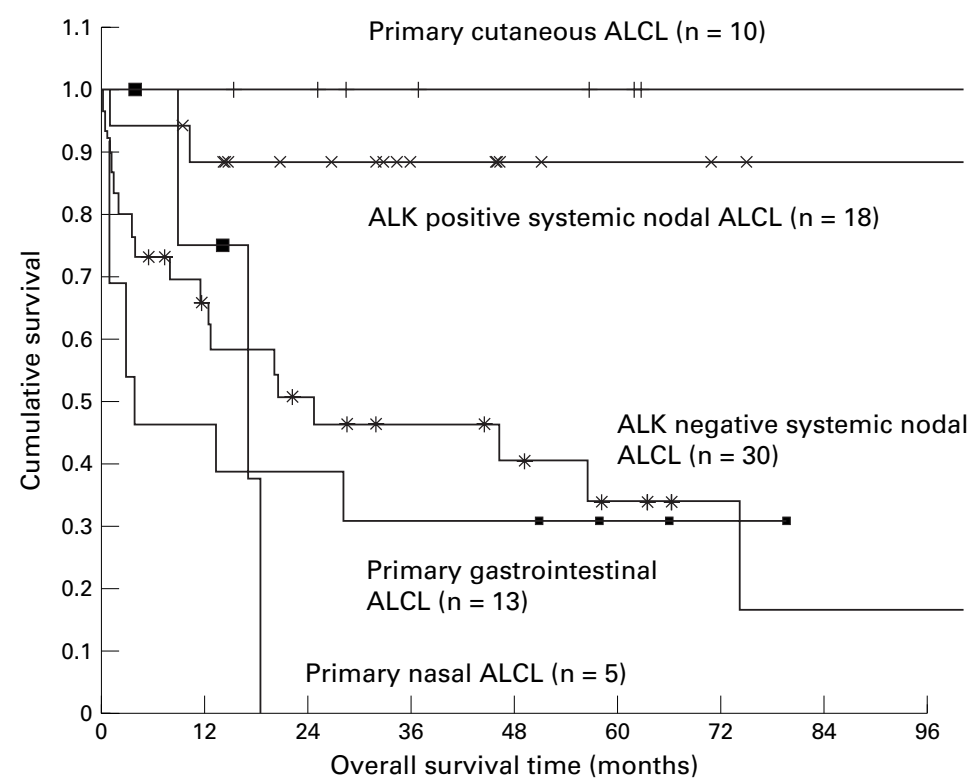

Figure 4 Comparison of overall survival time in anaplastic large cell lymphoma (ALCL), according to primary site of origin and anaplastic lymphoma kinase (ALK) expression. four of nine ${ }^{28}$ cutaneous ALCLs using nested RT-PCR analysis with two amplification steps. Because nested RT-PCR analysis is extremely sensitive, false positive results might have occurred. This seems probable in at least one study, ${ }^{27}$ where the cutaneous case showing amplification with nested RT-PCR failed to stain with the polyclonal anti-ALK antibody p80. In the other study, ${ }^{28}$ the cases positive for nested RT-PCR showed variable positivity with other detection methods, including p80 immunostaining and ISH. Yet another study ${ }^{29}$ found positive staining with the antibodies p80 and ALK1 in one of eight cases of primary cutaneous ALCL, but no evidence of ALK expression with standard RT-PCR.

Differentiating between systemic (nodal) and primary extranodal ALCL has consequences for therapeutic strategies. Of peripheral $\mathrm{T}$ cell lymphomas, systemic nodal ALCL has been shown to run a relatively favourable clinical course, ${ }^{4}$ whereas primary gastrointestinal and nasal $\mathrm{T}$ cell lymphomas usually show very aggressive behaviour. ${ }^{2-9}$ Indeed, when comparing clinical behaviour between ALCL arising at various sites, the site of origin was found to be strongly predictive of survival, with primary cutaneous ALCL showing excellent survival, whereas primary gastrointestinal and primary nasal ALCL were characterised by poor prognosis, comparable to ALK negative systemic nodal ALCL. Excluding primary cutaneous ALCL, the site of origin was not predictive of survival in ALK negative ALCL. Although the cases studied were relatively few, this suggests that in non-cutaneous ALCL, ALK expression rather than site of origin is important in predicting clinical outcome of the disease. Indeed, ALK expression predisposed to favourable clinical outcome, in accordance with previous studies. ${ }^{19-23}$ Interestingly, the patient presenting with concurrent intestinal and nodal (ALK positive) ALCL had a favourable clinical outcome, in contrast to primary gastrointestinal or nasal ALCL. Our findings support a growing opinion that ALK positive ALCL seems to be a distinct entity, characterised by good prognosis.

In patients who had multiple biopsies taken at initial diagnosis or who had recurrent disease, ALK expression was found to be consistent for all tumour biopsies-for example, if a patient was diagnosed with ALK positive ALCL, all other tumour biopsies of that patient proved ALK positive, regardless of localisation. For instance, the patient with concurrent intestinal and nodal ALCL showed ALK positivity in both localisations. Another patient with systemic (nodal) ALCL developed cutaneous and nodal relapses, all of which showed ALK expression. Vice versa, if the primary tumour was ALK negative, all other biopsies were found to lack ALK expression as well. The same staining pattern (cytoplasmic and nuclear, or cytoplasmic only) was observed for all biopsies of a given patient. These findings indicate that the chromosomal aberration leading to expression of the ALK protein is an early pathogenic event-before dissemination to other sites. In transfection experiments, ALK 
has been shown to have transforming abilities in vitro, ${ }^{33}{ }^{34}$ and to give rise to large B cell lymphomas in mice, ${ }^{35}$ but its precise role in the pathogenesis of ALCL remains to be elucidated.

The ALK1 and ALKc monoclonal antibodies gave identical results with regard to presence of staining, and localisation pattern of staining, the only difference being that ALKc showed stronger nuclear staining than ALK1. In 15 of 20 ALK positive cases, staining was observed in both the cytoplasm and nucleus of the tumour cells. In five of 20 cases, staining was restricted to the cytoplasm. This observation has been reported before in independent studies by other groups, ${ }^{11-13} 1730$ the hypothesis put forward being that these tumour cells carry translocations other than $t(2 ; 5)$, with the ALK gene linked to a gene other than NPM.

We conclude that ALK expression, if present in an extranodal ALCL site, favours systemic disease with (primary) nodal involvement and a good prognosis. Patients presenting with ALK positive extranodal ALCL should be carefully staged to determine whether lymph nodes are involved. ALK is expressed consistently in multiple and subsequent biopsies of a given patient, indicating that the chromosomal abnormality involving the ALK gene always occurs before dissemination to other sites. Finally, with the exception of a primary skin localisation, ALK negative ALCLs originating from different sites show comparable five year survival of less than $40 \%$. We recommend studies involving larger numbers of patients to confirm these findings.

1 Stein H, Mason DY, Gerdes J, et al. The expression of the Hodgkin's disease associated antigen $\mathrm{Ki}-1$ in reactive and neoplastic lymphoid tissue: evidence that Reed-Sternberg cells and histiocytic malignancies are derived from activated lymphoid cells. Blood 1985;66:848-58.

2 Harris NL, Jaffe ES, Stein H, et al. A revised EuropeanAmerican classification of lymphoid neoplasms: a proposal from the international lymphoma study group. Blood 1994; 84:1361-92.

3 de Bruin PC, Beljaards RC, van Heerde P, et al. Differences in clinical behaviour and immunophenotype between primary cutaneous and primary nodal anaplastic large cell lymphoma of T-cell or null cell phenotype. Histopathology 1993;23:127-35.

4 de Bruin PC, Noorduyn AL, van der Valk P, et al. Noncutaneous T-cell lymphomas. Recognition of a lymphoma type (large cell anaplastic) with a relatively favourable prognosis. (large cell anaplastic) with a

5 Willemze R, Kerl H, Sterry W, et al. EORTC classification for primary cutaneous lymphomas: a proposal from the cutaneous lymphoma study group of the European Organization for Research and Treatment of Cancer. Blood 1997;90:354-71.

6 Tilly $\mathrm{H}$, Gaulard P, Lepage E, et al. Primary anaplastic large-cell lymphoma in adults: clinical presentation, immunophenotype, and outcome. Blood 1997;90:3727-34.

7 Jaffe ES, Chan JKC, Su IJ, et al. Report of the workshop on nasal and related extranodal angiocentric T/natural killer cell lymphomas. Am F Surg Pathol 1996;20:103-11.

8 Sanchezbueno F, Garciamarcilla JA, Alonso JD, et al. Prognostic factors in primary gastrointestinal non-Hodgkins lymphomas-a multivariate analysis of 76 cases. Eur $\mathcal{F}$ Surg 1998;164:385-92.

9 Zinzani PL, Magagnoli M, Pagliani G, et al. Primary intestinal lymphoma-clinical and therapeutic features of 32 nal lymphoma-clinical and therapeutic

10 Morris SW, Kirstein MN, Valentine MB, et al. Fusion of a kinase gene, ALK, to a nucleolar protein gene, NPM, in kinase gene, ALK, to a nucleolar protein gene, NPM,
non-Hodgkin's lymphoma. Science 1994;263:1281-4.

11 Lamant L, Dastugue N, Pulford K, et al. A new fusion gene TPM3-ALK in anaplastic large cell lymphoma created by a $(1 ; 2)(\mathrm{q} 25 ; \mathrm{p} 23)$ translocation. Blood 1999;93:3088-95.
12 Wlodarska I, De Wolf-Peeters C, Falini B, et al. The cryptic inv(2) (p23q35) defines a new molecular genetic subtype of ALK-positive anaplastic large cell lymphoma. Blood

13 Mason DY, Pulford KAP, Bischof D, et al. Nucleolar localization of the nucleophosmin-anaplastic lymphoma kinase is not required for malignant transformation. Cancer Res 1998;58:1057-62.

14 Bischof D, Pulford K, Mason DY, et al. Role of nucleophosmin (NPM) portion of the non-Hodgkin's lymphomaassociated NPM-anaplastic kinase fusion protein in oncogenesis. Mol Cell Biol 1997;17:2312-25.

15 Pulford K, Lamant L, Morris SW, et al. Detection of anaplastic lymphoma kinase (ALK) and nucleolar protein nucleophosmin (NPM)-ALK proteins in normal and neoplastic cells with the monoclonal antibody ALK1. Blood plastic cells with the

16 Lamant L, Meggetto F, Al Saati T, et al. High incidence of the $\mathrm{t}(2 ; 5)(\mathrm{p} 23 ; \mathrm{q} 35)$ translocation in anaplastic large cell ymphoma and its lack of detection in Hodgkin's disease: comparison of cytogenetic analysis, reverse transcriptasepolymerase chain reaction, and p80 immunostaining. Blood 1996;87:284-91.

17 Falini B, Bigerna B, Fizzotti M, et al. ALK expression defines a distinct group of T/null lymphomas ("ALK lymphomas") with a wide morphological spectrum. Am f Pathol 1998;153:875-86.

18 Shiota $M$, Takenaga $M$, Satoh $\mathrm{H}$, et al. Diagnosis of $\mathrm{t}(2 ; 5)(\mathrm{p} 23 ; \mathrm{q} 35)$-associated Ki-1 lymphoma with immunohistochemistry. Blood 1994;84:3648-52.

19 ten Berge RL, Dukers DF, Oudejans JJ, et al. Adverse effects of activated cytotoxic T-lymphocytes on the clinical outcome of nodal anaplastic large cell lymphoma. Blood 1999;93:2688-96.

20 Sandlund JT, Pui $\mathrm{CH}$, Roberts $\mathrm{M}$, et al. Clinicopathologic features and treatment outcome of children with large-cell lymphoma and the $\mathrm{t}(2 ; 5)(\mathrm{p} 23 ; \mathrm{q} 35)$. Blood 1994;84:246771

21 Shiota M, Nakamura S, Ichinoshama R, et al. Anaplastic large cell lymphomas expressing the novel chimeric protein p80 NPM/ALK: a distinct clinicopathologic entity. Blood 1995;86:1954-60.

22 Falini B, Pileri S, Zinzani PL, et al. ALK-positive lymphoma: clinicopathological findings and outcome. Blood 1999;93:2697-706.

23 Gascoyne RD, Aoun P, Wu D, et al. Prognostic significance of anaplastic lymphoma kinase (ALK) protein expression in adults with anaplastic large cell lymphoma. Blood 1999; 93:3913-21.

24 Herbst H, Sander C, Tronnier M, et al. Absence of anaplastic lymphoma kinase (ALK) and Epstein-Barr virus gene products in primary cutaneous anaplastic large cell lymphoma and lymphomatoid papulosis. $\mathrm{Br} f$ Dermatol 1997;137:680-6.

25 DeCoteau JF, Butmarc JR, Kinney MC, et al. The t(2;5) chromosomal translocation is not a common feature of primary cutaneous CD30+ lympoproliferative disorders: comparison with anaplastic large-cell lymphoma of nodal origin. Blood 1996;87:3437-41.

26 Pittaluga S, Wlodarska I, Pulford K, et al. The monoclonal antibody ALK1 identifies a distinct morphological subtype of anaplastic large cell lymphoma associated with 2p23/ ALK rearrangements. Am f Pathol 1997;151:343-51.

27 Ott G, Katzenberger T, Siebert R, et al. Chromosomal abnormalities in nodal and extranodal CD30+ anaplastic large cell lymphomas - infrequent detection of the $t(2 ; 5)$ in extranodal lymphomas. Genes Chromosomes Cancer 1998; 22:114-21

28 Beylot-Barry M, Lamant L, Vergier B, et al. Detection of $\mathrm{t}(2 ; 5)(\mathrm{p} 23 ; \mathrm{q} 35)$ translocation by reverse transcriptase polymerase chain reaction and in situ hybridization in CD30-positive primary cutaneous lymphoma and lymphomatoid papulosis. Am f Pathol 1996;149:483-92.

29 Su LD, Schnitzer B, Ross CW, et al. The t $(2 ; 5)$-associated p80 NPM/ALK fusion protein in nodal and cutaneous CD $30+$ lymphoproliferative disorders. f Cutan Pathol 1997;24:597-603.

30 Benharroch D, Meguerian-Bedoyan Z, Lamant L, et al. BLK-positive lymphoma: a single disease with a broad ALK-positive lymphoma: a single disease with a
spectrum of morphology. Blood 1998;91:2076-84.

31 Kinney MC, Collins RD, Greer JP, et al. A small cell predominant variant of primary Ki-1 (CD30)+ T-cell lymphoma. Am $\mathcal{F}$ Surg Pathol 1993;17:859-68.

32 Bobrow MN, Shaughnessy KJ, Litt GJ. Catalysed reporter deposition: a novel method of signal amplification. II. Application to membrane immunoassays. F Immunol Methods 1991;137:103-12.

33 Fujimoto J, Shiota M, Iwahara T, et al. Characterization of the transforming activity of $\mathrm{p} 80$, a hyperphosphorylated protein in a $\mathrm{Ki}-1$ lymphoma cell line with chromosomal translocation t(2;5). Proc Natl Acad Sci US A 1996;93:4181-6.

34 Wellmann A, Doseeva V, Butscher W, et al. The activated anaplastic lymphoma kinase increases cellular proliferation and oncogene up-regulation in rat $1 \mathrm{~A}$ fibroblasts. FASEB $\mathcal{F}$ 1997;11:965-72

35 Kuefer MU, Look AT, Pulford K, et al. Retrovirus-mediated transfer of NPM-ALK causes lymphoid malignancy in mice. Blood 1997;90:2901-10. 